\title{
AVALIAÇÃO RETROSPECTIVA DO SERVIÇO AMBULATORIAL EM DIETOTERAPIA DO HOSPITL DAS CLÍNICAS DA UNIMERSIDADE FEDERAL DE GOIÁS
}

\section{RETROSPECTIVE EVALUATION OF THE THERAPY AMBULATORY SERVICE AT THE HOSPITAL DAS CLÍNICAS OF THE UNMERSIDADE FEDERAL DE GOIÁS - BRAZIL}

\author{
Maria Luiza Ferreira STRINGHINI \\ Gersislei Antonia SALADO' \\ Joaquim Tomé de SOUSA ${ }^{2}$ \\ Márcia Armentano Clark REIS \\ Adriane Cecília Teixeira Oliveira TELES ${ }^{1}$
}

\section{RESUMO}

A proposta deste estudo foi fazer uma retrospectiva sobre o atendimento do Serviço Ambulatorial de Nutrição do Hospital das Clinicas da Universidade Federal de Goiás de 1991 a 1995. A finalidade do ambulatório é prestar orientação nutricional a indivíduos sadios e enfermos da comunidade bem como aos funcionários e estudantes da Universidade. $O$ atendimento é feito com base em registros em fichas individuais, com um rigoroso controle de medidas de peso e altura, além de um recordatório alimentar de 24 horas e um registro de freqüência de ingestão. A partir dai, faz-se a prescrição de dieta, que é individualizada, levando-se em conta as condições socioeconômicas e culturais do cliente. Na análise retrospectiva foram consultados os prontuários nutricionais e avaliados os dados referentes à idade, ao sexo, à classificação do estado nutricional, ao diagnóstico médico, a doenças associadas e ao número de consultas de retorno. No período foram atendidos 646 pacientes sendo 26,2\% do sexo masculino e 73,8\% do sexo feminino, dos quais 24,7\% se encontravam na faixa etária de 19 a 35 anos. As principais doenças encontradas foram obesidade (50,8\%) e diabetes (33,3\%). Quanto à classificação do estado nutricional, 33,9\% dos indivíduos diabéticos apresentavam sobrepeso e 30,2\% eram eutróficos. Entre os pacientes cuja queixa principal era obesidade, 68,3\% eram realmente classificados como obesos. Obsevou-se que 40,9\% dos clientes diabéticos possuíam outras doenças associadas sendo este número de 44,5\% entre os obesos. Entretanto, $61 \%$ dos pacientes atendidos não retornaram às consultas. Portanto, não se deve esquecer da importância de uma avaliação constante do serviço de nutrição ambulatorial que é oferecido à comunidade, a fim de melhorar a eficiência do tratamento e oferecer subsídios para o planejamento de novas atividades.

Termos de indexação: avaliação nutricional, dietoterapia, serviços de saúde comunitária, pacientes ambulatorias, serviço hospitalar de nutrição.

\footnotetext{
(1) Professoras do Departamento de Nutrição da Faculdade de Enfermagem e Nutrição da Universidade Federal de Goiás.

(2) Professor do Departamento de Enfermagem da Faculdade de Enfermagem e Nutrição da Universidade Federal de Goiás.
} 


\begin{abstract}
The aim of this paper is to evaluate, in a restropective analysis, the attendance at the nutrition ambulatory service of the Hospital das Clinicas of the Universidade Federal de Goiás, Brazil, from 1991 to 1995. The purpose of the ambulatory is to give nutritional orientation to healthy and sick people of the community, and also to the employees and the students of the University. The attendance is done with the use of an individual register that includes a rigorous control of weight and height data, a 24 hours record of diet and behaviour information and a record of the eating frequency. The diet prescription is individual and it is based on the cultural, social and economic conditions of the patient. In the retrospective analysis, nutritional forms were consulted, and data about age, sex, nutritional status, medical diagnosis, associated diseases and number of returning consultations were evaluated. It was observed that 646 patients were attended, of which $26.2 \%$ were male and $73.8 \%$ were female, and $24.7 \%$ of them aged 19 to 35 years. The main diseases verified were obesity (50.8\%) and diabetes (33.3\%). Concerning to nutritional status classification, $33.9 \%$ of the diabetic clients were overweight and $30.2 \%$ were eutrophic. Among the patients whose main complaint was obesity, $68.3 \%$ were really classified as obese. It was observed that 40.9\% \of the diabetic patients and $44.5 \%$ of the obese clients showed other diseases associated. However, $61.0 \%$ of the patients did not return to the next consultation. Therefore, it must be remembered that a constant evaluation of the nutrition ambulatory service that is offered to the community is important, in order to increase the efficiency of the dietetic treatment and to support the planning of the constant reformulation of the activities.
\end{abstract}

Index terms: nutritional assesment, diet therapy, community health .services, outpatients, food service hospital.

\section{INTRODUÇÃO}

O Serviço Ambulatorial em Dietoterapia do Hospital das Clínicas da Universidade Federal de Goiás (HC/UFG) foi implantado em 1991, com a idade de prestar orientação nutricional a indivíduos sadios e enfermos, implementando assim um sistema de vigilância nutricional visando a prevenção de doenças e a promoção da saúde.

A UFG é conveniada ao Sistema Unificado de (SUS) e presta atendimento ambulatorial gratuito nos setores médico, odontológico, psicológico, fisioterápico, de assistência social e nutricional, que é assegurado a seus funcionários, estudantes, docentes e a toda população do município de Goiânia e cidades vizinhas, tendo atendido em 1995, 275230 pessoas.

As metas principais das atividades do ambulatório de Nutrição do HC/UFG são:

1. Orientar a terapêutica dietética de clientes externos e internos encaminhados pelo serviço médico,

2. Divulgar para a comunidade os princípios básicos de nutrição necessários à promoção da saúde,
3. Viabilizar feedback contínuo para reformulação e ampliação do ensino na área de Nutrição, através de estágio promovido aos alunos do $5^{\circ}$ ano de Nutrição (ROSADO et al.,1993),

4. Integrar a equipe de profissionais da saúde do $\mathrm{HC} / \mathrm{UFG}$,

5. Divulgar a atuação do profissional nutricionista em consultórios e ambulatórios.

A regulamentação da profissão de nutricionista, através da Lei $n^{\circ}$ 5276/67 (BRASIL,1967) permitiu a este profissional tornar-se integrante das equipes de saúde pública e hospitalares. O nutricionista tornou-se mais conhecido e passou gradativamente a expandir suas ações de cuidado hospitalar a clientes externos, através de atendimento ambulatorial ${ }^{3}$.

A proposta deste estudo foi de fazer uma avaliação retrospectiva sobre o atendimento de um serviço ambulatorial de nutrição no HC/LTFG de 1991 a 1995, com o objetivo de avaliar o serviço prestado à comunidade, por meio do conhecimento do perfil dos pacientes atendidos no ambulatório, bem como elaborar

\footnotetext{
(3) A Profissão recebeu nova regulamentação em setembro de 199I, pela Lei no 8234 (Nota do Conselho Editorial).
} 
propostas para melhorar e ampliar o nível do atendimento.

\subsection{Rotina de atendimento}

$\mathrm{O}$ atendimento nutricional é efetuado quando indivíduos sadios solicitam espontaneamente orientação nutricional, ou para correção de excesso de peso, bem como por encaminhamento médico para os portadores de doenças, que necessitem de dietas específicas. As primeiras consultas tem duração média de 60 minutos e, as consultas de retorno, aproximadamente 20 minutos.

Para o cadastramento do cliente utiliza-se um prontuário específico onde constam seus dados biopsicossociais, além do prontuário médico. Após este registro, procede-se à anamnese alimentar utilizando-se o questionário de freqüência e o recordatório alimentar de 24 horas conforme preconizado por ANSELMO et al. (1992) e DIEKEN (1992).

$\mathrm{Na}$ avaliação do estado nutricional são considerados também os resultados dos testes bioquímicos e antropométricos. $\mathrm{O}$ exame físico é importante, principalmente em crianças e adolescentes, para avaliação do peso, do crescimento e do seu desenvolvimento. A classificação do grau de nutrição é feita pela tabela de percentil do National Center for Health Statistics (ORGANIZACIÓN...,1983) e para indivíduos adultos, o peso ideal é determinado pelo Índice de Massa Corporal (IMC) de acordo com o proposto por FERREIRA (1986) e WAITZBERG \& FERRINI (1995).

A dietaé individualmente planejada, respeitando hábitos alimentares pessoais, as preferências, assim como o nível socioeconômico. O paciente recebe a dieta no final da consulta, em impresso próprio, onde as quantidades dos alimentos são fornecidas em medidas caseiras, com orientação sobre eventuais substituições através da equivalência alimentar.

Para o acompanhamento do paciente são marcadas consultas de retorno após duas a três semanas, para reavaliar a dieta prescrita ou esclarecer as dúvidas relacionadas à programação dietética adotada. Quando o paciente atinge os objetivos propostos no início do tratamento, recebe alta com a orientação nutricional necessária.

\section{MATERIAL E MÉTODOS}

O levantamento retrospectivo dos clientes atendidos desde o início das atividades ambulatoriais foi feito através da análise dos prontuário nutricionais dos anos de 1991 a 1995.

Foram avaliados os seguintes parâmetros: sexo, idade, Índice de Massa Corporal (IMC), diagnóstico médico, doenças associadas e número de consultas de retorno.

Os diagnósticos foram agrupados segundo a queixa principal do paciente, e classificados em três problemas básicos de saúde: a) diabetes; b) obesidade c) outros, sendo que neste último estão contidas alterações do trato gastrintestinal, cardiorrespiratório e renal. Posteriormente, a classificação do estado nutricional foi realizada pelo Índice de Quetelet nas seguintes categorias: desnutrido - menor ou igual a 15 $\mathrm{kg} / \mathrm{m}^{2}$; baixo peso - de $15,1 \mathrm{a} 18 \mathrm{~kg} / \mathrm{m}^{2}$; eutróficos - de 18,1 a $25 \mathrm{~kg} / \mathrm{m}^{2}$; sobrepeso - de 25,1 a $27 \mathrm{~kg} / \mathrm{m}^{2}$ e obeso - maior ou igual a $27,1 \mathrm{~kg} / \mathrm{m}^{2}$ (BLACKBURN et al., 1977). Para a classificação do grau de nutrição de crianças e adolescentes foi usada a tabela de percentil para peso/altura do National Center for Health Statistics (ORGANIZACIÓN..., 1983), sendo que os indivíduos foram considerados desnutridos quando o percentil era menor que 3; de baixo peso para faixa entre 3 e 10; (eutróficos entre 10 e 90; de sobrepeso entre 90 e 95 e obesos para valores de percentil acima de 95 .

Os tratamentos estatísticos utilizados foram a análise percentual e de freqüência, através do programa estatístico Epi Info (DEAN et al.,1990).

\section{RESULTADOS E DISCUSSÃO}

De 1991 a 1995 foram atendidos no ambulatório nutricional 646 indivíduos, distribuídos por ano. Analisando a Tabela 1, observou-se um aumento gradual no número de indivíduos atendidos anualmente. Em 1994, houve uma diminuição no número de atendimentos em decorrência de uma paralisação nos serviços prestados à comunidade para reformulação das atividades ambulatoriais. 
Tabela 1. Indivíduos atendidos no Serviço Ambulatorial de Nutrição da UFG de acordo com o ano.

\begin{tabular}{lcc}
\hline Ano & \multicolumn{2}{c}{ Indivíduos atendidos } \\
\hline & $\mathrm{n} \cong$ & $\%$ \\
1991 & 68 & 10,6 \\
1992 & 88 & 13,6 \\
1993 & 144 & 22,3 \\
1994 & 128 & 19,8 \\
1995 & 218 & 33,7 \\
\hline Total & 646 & 100,0 \\
\hline
\end{tabular}

Observou-se que dos 646 pacientes atendidos, $169(26,2 \%)$ eram do sexo masculino e $477(73,8 \%)$ eram do sexo feminino, atestando uma alta prevalência para indivíduos do sexo feminino neste serviço. SAMPAIO \& SOUZA (1991) em trabalho similar, observaram igualdade de procura pelos dois sexos no serviço ambulatorial de nutrição em Fortaleza, Ceará.

Houve grande procura pelo serviço de nutrição por indivíduos na faixa etária que varia de 19 a 35 anos $(27,4 \%)$, seguidos com 51 a 65 anos $(24,5 \%)$ e com 36 a 50 anos $(24,3 \%)$ (Tabela 2$)$.

Tabela 2. Distribuição de indivíduos quanto à faixa etária.

\begin{tabular}{lcc}
\hline Idade (anos) & \multicolumn{2}{c}{ População } \\
\hline & no & $\%$ \\
1 a 12 & 81 & 12,5 \\
13 a 18 & 34 & 5,3 \\
19 a 35 & 177 & 27,4 \\
36 a 50 & 157 & 24,3 \\
51 a 65 & 158 & 24,5 \\
$>$ 65 & 39 & 6,0 \\
\hline Total & 646 & 100,0 \\
\hline
\end{tabular}

As doenças existentes na clientela atendida estão mostradas na Tabela 3, sendo que alguns indivíduos eram acometidos por mais de um problema de saúde, mas para fins estatísticos computou-se a queixa principal, razão do encaminhamento ao nutricionista(SGAVIOLI,1993a).

Tabela 3. Distribuição de indivíduos quanto à queixa principal.

\begin{tabular}{lcc}
\hline Doenças & \multicolumn{2}{c}{ População } \\
\hline & $\mathrm{n}^{\circ}$ & $\%$ \\
Diabetes mellitus & 215 & 33,3 \\
Obesidade & 328 & 50,8 \\
Outros & 103 & 15,9 \\
\hline Total & 646 & 100,0 \\
\hline
\end{tabular}

Observou-se uma grande prevalência de clientes obesos atendidos $(50,8 \%)$. Tal prevalência ocorre tanto nas camadas de nível socioeconômico mais alto quanto nas demais, determinando sérios riscos para a saúde, como a associação com o diabetes, as doenças cardiovasculares e as osteoartroses. Conforme DIDIO (1995) mais de um terço de todos os atendimentos em nível ambulatorial e hospitalar é ocupado pela obesidade e suas complicações, o que sobrecarrega a rede de atendimento da Saúde Pública.

Pelos resultados obtidos observou-se que dos 215 indivíduos diabéticos atendidos, 40,9\% apresentavam outras doenças associadas, sendo que $25 \%$ eram doenças cardiovasculares. Para os obesos, $44,5 \%$ apresentavam outras doenças associadas, sendo que $61,6 \%$ eram cardiovasculares.

A Figura 1 mostra a distribuição do estado nutricional dos indivíduos atendidos. Observou-se entre os 215 diabéticos não foram encontrados desnutridos e, apenas 7,0\% encontravam-se com baixo peso, $28,0 \%$ foram considerados obesos, $30,2 \%$ eram eutróficos e $33,9 \%$ apresentavam sobrepeso, tendo portanto procurado o nutricionista para orientação e controle da dieta. Verificou-se também que dos 328 indivíduos cuja queixa principal era obesidade, $4,9 \%$ não apresentavam aumento de peso, 26,8\% apresentavam sobrepeso, mas a maioria, $68,3 \%$, era realmente composta por obesos. Entre os portadores de outras doenças, verificou-se que $53,4 \%$ eram eutróficos, tendo procurado o serviço para orientação nutricional. Observou-se também que neste grupo, $6,8 \%$ eram desnutridos, $17,5 \%$ apresentavam baixo peso, $15,5 \%$ foram classificados como sobrepeso e apenas $6,8 \%$ eram obesos.

Uma forma de se avaliar a receptividade do paciente à orientação dietoterápica é a sua freqüência na reconsulta (MOURA et al.,1982; SAMPAIO \& SOUZA, 1991; SGAVIOLI, 1993a; SGAVIOLI, 1993b). Neste Serviço verificou-se que $61,0 \%$ dos pacientes atendidos não retornaram às próximas consultas e do total de consultas de retorno, $52,8 \%$ retornaram uma vez, $25,0 \%$, duas vezes e $22,2 \%$, de três a dez vezes. SAMPAIO \& SOUZA (1991) observaram um abandono do tratamento em 20,4\% dosindivíduos, portanto torna-senecessáriauma análise crítica deste serviço devido ao grande índice de clientes que não prosseguiram no tratamento. 


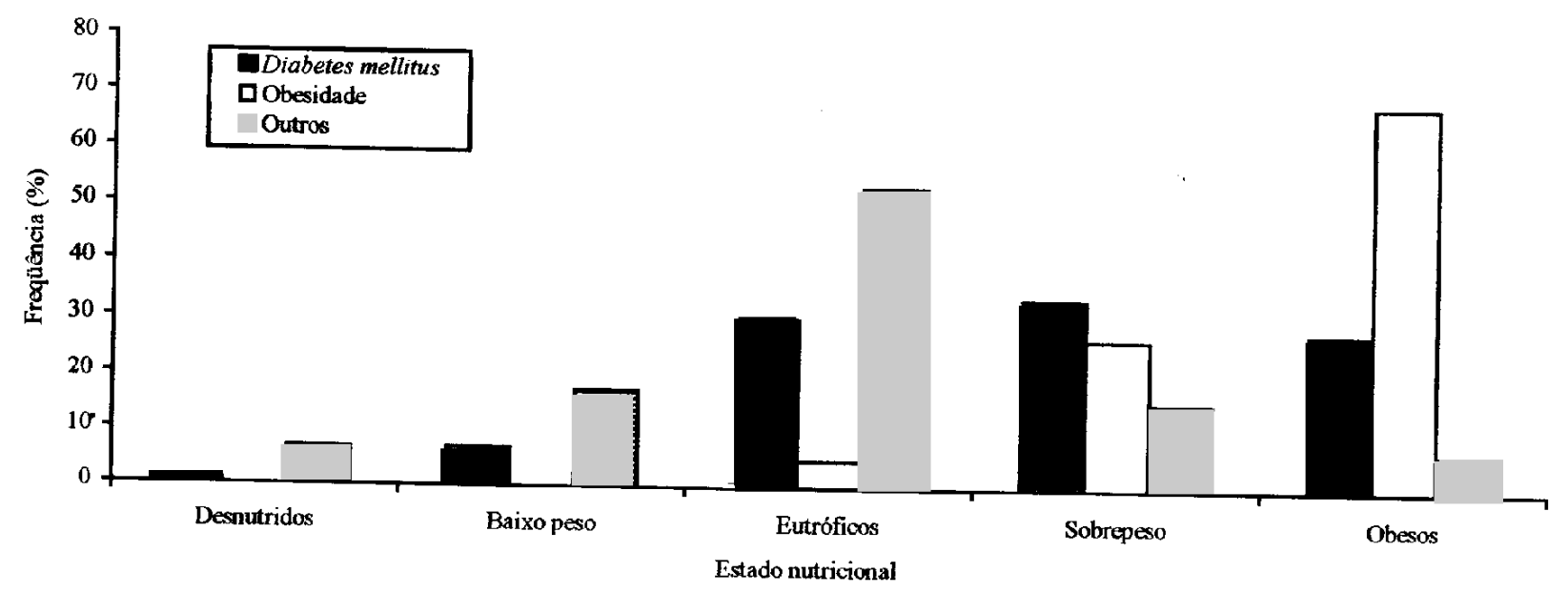

Figura 1. Distribuição dos indivíduos quanto à classificação do estado.

Vários problemas podem estar relacionados ao abandono do tratamento. Uma primeira dificuldade a ser relacionada pode estar ligada ao manuseio inadequado, pelo paciente, da lista de substituições, o que o levaria a considerar as orientações e as dietas restritas. Além disso, muitas vezes a primeira consulta pode ser super valorizada pelo nutricionista oferecendo aos pacientes uma grande carga de informações que não são devidamente trabalhadas nas consultas posteriores. Outro ponto a ser considerado seria o enfoque técnico exagerado na orientação e nos cálculos da dieta que muitas vezes não considera o histórico de vida do indivíduo, impondo conhecimentos sem discutir as possibilidades e alternativas alimentares (ZELMANOWICZ \& DELLANDA, 1992; SGAVIOLI, 1993a).

A efetividade das medidas dietéticas adotadas no Serviço Ambulatorial de Nutrição do HC/UFG é evidenciada pela variação da classificação do grau de nutrição dos pacientes, calculada a partir de medidas antropométricas realizadas na primeira consulta e nas reconsultas. Observa-se na Figura 2 que a porcentagem de indivíduos classificados como desnutridos e de baixo peso diminuiu ao final do tratamento, enquanto a porcentagem de pacientes eutróficos aumentou, indicando recuperação nutricional e efetividade no tratamento. Pode-se ainda inferir que o aumento na porcentagem de indivíduos eutróficos deveu-se à mudança do estado nutricional dos indivíduos desnutridos e de baixo peso, ou mesmo pela reclassificação daqueles com sobrepeso, ou até mesmo obesos, que tiveram êxito ao final do tratamento.
Com relação às alterações observadas na Figura 2, verificou-se aumento na porcentagem de indivíduo; que apresentavam sobrepeso; é provável que tenha ocorrido, pois os indivíduos, anteriormente classificados como obesos evoluíram para a classe de sobrepeso com o transcorrer do tratamento. Percebeu-se ainda que a porcentagem de indivíduos obesos diminuiu com as medidas nutricionais adotadas, embora ainda tenha permanecido alta. Resultados similares foram observados por SGAVIOLI ( 1993b) no Serviço Ambulatorial de Obesos no Hospital Universitário da UFRJ.

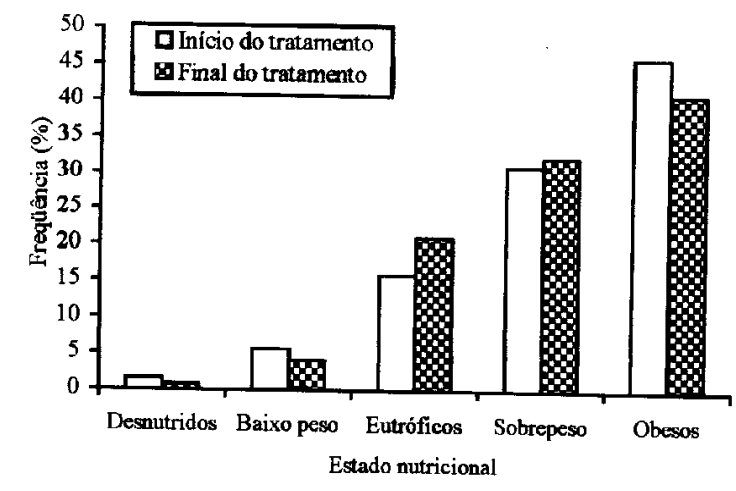

Figura 2. Distribuição dos indivíduos de acordo com a classificação do estado nutricional no início e no final do tratamento.

\section{CONCLUSÃO}

Ao constatar a alta taxa de abandono do tratamento verificado no Serviço Ambulatorial de 
Nutrição do HC/UFG, torna-se necessária uma reformulação do atendimento oferecido e dos objetivos a serem atingidos. Assim sendo, é possível propor algumas medidas que minimizem as dificuldades enfrentadas na prática profissional, tornando mais eficaz a manutenção e/ou recuperação da saúde da clientela assistida. Para tanto recomenda-se:

1. Que o nutricionista faça um programa de acompanhamento nutricional adequado, utilizando técnicas de mudança de comportamento do paciente, por exemplo reuniões de grupo e utilização de vídeos educativos conforme proposto por CARSON \& HASSEL (1994) e SOWINSKI et al. (1994),

2. Que exista entrosamento com outros profissionais que acompanham o cliente, para que sejam tomadas decisões comuns durante o tratamento de acordo com REBOVICH et al. (1994) e

3. Que o nutricionista revise periodicamente os instrumentos e padrões utilizados durante a consulta.

Portanto, não se deve esquecer da importância de uma avaliação constante do serviço de nutrição ambulatorial que são oferecidos à comunidade, para melhorar a eficiência do tratamento e fornecer subsídios para o planejamento de novas atividades.

\section{REFERÊNCIAS BIBLIOGRÁFICAS}

ANSELMO, M.A.C., BURINI, R.L., ANGELELI, A.Y.O., MOTA, N.G.S., CAMPANA, A.O. Avaliação do estado nutricional de indivíduos adultos sadios de classe média: ingestão energética e protéica, antropometria, exames bioquímicos do sangue e testes de imunocompetência. Revista de Saúde Pública, São Paulo, v.26, n.1, p.46-53, 1992.

BLACKBURN, G.L., BISTRIAN, B.R., MAINI, B.S., SCHLAMM, H.T., SMITH, M.F. Nutritional and metabolic assessment of the hospitalized patient. Journal of Parenteral and Enteral Nutrition, Baltimore, v.1,n.1,p.11-22, 1977.

BRASIL. Lei n.5276, de 24 de abril de 1967. Dispõe sobre a profissão de nutricionista, regulamenta seu exercício e dá outras providências. Diário Oficial [da República Federativa do Brasil], Brasília, p. 4707, 26 abr 1967.

CARSON, C.A., HASSEL, C.A. Educating high-risk Minnesotans about dietary fats blood cholesterol and heart disease. Journal of the American Dietetic Association, Chicago, v.94, n.6, p.659-660,1994.
DEAN,A.G,DEAN,J.A.,BURTON,A.H.,DICKER,R.C.Epi Info version $5.01 \mathrm{~b}$ : a word processing, database, and statistics program for epidemiology on microcomputers. Atlanta : Center for Disease Control,1990.367p.

DIDIO, R. Princípios básicos para a terapêutica da obesidade. Consenso da Associação Brasileira para Estudo da Obesidade. Arquivos Brasileiros de Endocrinologia e Metabologia, São Paulo, v.39, n.3/4, p.202-204,1995.

DIEKEN, H.A. Educação nutricional. In: KRAUSE, M.V., MAHAN, L.K. Alimentos, nutrição e dietoterapia. 7.ed. São Paulo : Roca,1992.p.373.

FERREIRA,M.C., VEIGA, G.V.da,MARCHIM,J.S.Estudo nutricional de pacientes do Hospital Universitário Júlio Müller, Cuiabá-MT. A Folha Médica, Rio de Janeiro, v.93,n.4,p.217-219,1986.

MOURA,E.C.,LEONE,A.L.,BENTO,A.L.G.,LEONE,C.A.L., SOUZA, L.C., NEVES, J.F., IMPROTA, M.T.W., MILIORINI,M., SORIANO, R. BIANCHIN, S. Avaliação de um serviço básico de saúde: uma abordagem comunitária. Revista de Nutrição da PUCCAMP, Campinas, v.5,n.2,p.101-120,1992.

ORGANIZACIÓN MUNDIALDELA SALUD. Medición del cambio del estado nutricional. Genebra, 1983. $105 \mathrm{p}$.

REBOVICH, E.J., WODARRSKI, L.A., HURLEY, R.S., GREENHALGH, S.R., STOMBAUGH,I.A. Auniversity-community model for the integration of nutrition research, practice and education. Journal of the American Dietetic Association, Chicago, v.94, n.2, p.179-182,1994.

ROSADO, L.E.F.P.L., ROSADO, G.P., RIBEIRO, S.M.R., MONTEIRO, J.B.R. O serviço de dietoterapia na divisão de saúde da Universidade Federal de Viçosa (UFV): implantação e funcionamento. Revista de Nutrição da PUCCAMP, Campinas, v. 6, n. 2, p. 133-158,1993.

SAMPAIO, H.A.C., SOUZA, A.M.H. Atuação do nutricionista em consultório: experiência de oito anos em Fortaleza-CE. Revista de Nutrição da PUCCAMP, Campinas, v.4,n.42,p.25-39,1991.

SGAVIOLI, M.E.A. Avaliação crítica da relação paciente/profissional em um hospital de ensino. Revista de Nutrição da PUCCAMP, Campinas, v.6, n. I, p.52-76, 1993a.

Custos do tratamento ambulatorial de obesos no Hospital Universitário da Universidade Federal do Rio de Janeiro (UFRJ).Revista de Nutrição da PUCCAMP, Campinas, v.6, n.2,p.159-183,1993b. 
SOWINSKI, S.A., SHEPHERD, S.K., DOWLING, R.A., WAGNER, N.H. Value-added services that increase physicians intent to refer patients to an outpatient nutrition clinic. Journal of the American Dietetic Association, Chicago, v.94, n.5, p.529-532,1994.

WAITZBERG, D.L., FERRINI, N.T. Avaliaçãonutricional. In: Nutrição enteral e parenteral napráticamédica. 2.ed. SãoPaulo:Atheneu 1995.p.127.
ZELMANOWICZ, A.M., DELLANDA, L.C. A consulta ambulatorial. In: DUNCAN, B.B., SCHMIDT, M.I., GIUGLIANI, E.R.J. Medicina ambulatorial. 3.ed. Porto Alegre: Artes Médicas,1992.p.31-34.

Recebido para publicação em 20 de agosto de 1996 e aceito em 11 de março de 1997. 